\title{
On the smallest simultaneous power nonresidue modulo a prime
}

\author{
K. Ford, M. Z. Garaev and S. V. Konyagin
}

\begin{abstract}
Let $p$ be a prime and $p_{1}, \ldots, p_{r}$ be distinct prime divisors of $p-1$. We prove that the smallest positive integer $n$ which is a simultaneous $p_{1}, \ldots, p_{r}$-power nonresidue modulo $p$ satisfies

$$
n<p^{1 / 4-c_{r}+o(1)} \quad(p \rightarrow \infty)
$$

for some positive $c_{r}$ satisfying $c_{r} \geqslant e^{-(1+o(1)) r}(r \rightarrow \infty)$.
\end{abstract}

\section{Introduction}

Let $n(p)$ be the smallest positive quadratic nonresidue modulo $p$ and $g(p)$ be the smallest positive primitive root modulo $p$. The problem of upper bound estimates for $n(p)$ and $g(p)$ starts from the early works of Vinogradov. It is believed that $n(p)=p^{o(1)}$ and $g(p)=p^{o(1)}$ as $p \rightarrow \infty$. Vinogradov [14, 15] proved that

$$
n(p) \ll p^{\frac{1}{2 \sqrt{e}}}(\log p)^{2}, \quad g(p)<\frac{2^{k+1}(p-1) p^{\frac{1}{2}}}{\phi(p-1)},
$$

where $k$ is the number of distinct prime divisors of $p-1$. Hua [9] improved Vinogradov's result to $g(p)<2^{k+1} p^{1 / 2}$ and then Erdös and Shapiro [6] refined it to $g(p) \ll k^{C} p^{\frac{1}{2}}$, where $C$ is an absolute constant. These bounds were improved by Burgess [1, 2] to

$$
n(p)<p^{\frac{1}{4 \sqrt{e}}+o(1)}, \quad g(p)<p^{\frac{1}{4}+o(1)} \quad(p \rightarrow \infty) .
$$

The Burgess bounds remains essentially the best known up to date, in a sense that it is not even known that $n(p) \ll p^{1 / 4 \sqrt{e}}$ or that $g(p) \ll p^{1 / 4}$. 
If one allows a small exceptional set of primes, then better estimates may be obtained. Using his "large sieve", Linnik [12] proved that for any $\varepsilon>0$, there are only $O_{\varepsilon}(\log \log x)$ primes $p \leqslant x$ for which $n(p)>p^{\varepsilon}$. The sharpest to date results for $g(p)$ (which also hold for the least prime primitive root modulo $p$ ) are due to Martin [13], who proved that for any $\varepsilon>0$, there is a $C>0$ so that $g(p)=O\left((\log p)^{C}\right)$ with at most $O\left(x^{\varepsilon}\right)$ exceptions $p \leqslant x$. All of these type of results are "purely existential", in that one cannot say for which specific primes $p$ the bounds hold (say, in terms of the factorization of $p-1)$.

From elementary considerations it follows that an integer $g$ is a primitive root modulo $p$ if and only if for any prime divisor $q \mid p-1$ the number $g$ is a $q$-th power nonresidue modulo $p$. Thus, if $p_{1}, \ldots, p_{k}$ are all the distinct prime divisors of $p-1$, then $g(p)$ is the smallest positive simultaneous $p_{1}, \ldots, p_{k}$-th power nonresidue modulo $p$. In the present paper we prove the following result.

Theorem 1. Let $p$ be a prime number and $p_{1}, \ldots, p_{r}$ be distinct prime divisors of $p-1$. Then the smallest positive integer $n$ which is a simultaneous $p_{1}, \ldots, p_{r}$-th power nonresidue modulo $p$ satisfies

$$
n<p^{1 / 4-c_{r}} e^{C(\log r)^{1 / 2}(\log p)^{1 / 2}}
$$

where $C>0$ is an absolute constant and $c_{r} \geqslant e^{-(1+o(1)) r}$ as $r \rightarrow \infty$.

The novelty of the result is given by the factor $p^{-c_{r}}$. We observe that for $c_{r}<(\log p)^{-1 / 2}$ (in particular, for $r \geqslant(0.5+\varepsilon) \log \log p$ and $p \geqslant p(\varepsilon)$ ) this factor is dominated by the exponential factor.

The following corollaries directly follow from Theorem 1.

Corollary 1. Let $p$ be a prime number and $p_{1}, \ldots, p_{r}$ be distinct prime divisors of $p-1$, where $r$ is fixed. Then the smallest positive integer $n$ which is a simultaneous $p_{1}, \ldots, p_{r}$-th power nonresidue modulo $p$ satisfies

$$
n<p^{1 / 4-c_{r}+o(1)} \quad(p \rightarrow \infty) .
$$

From our earlier discussion, the upper bound given in Theorem 1 holds also for $g(p)$ whenever $p-1$ has $r$ distinct prime factors.

Corollary 2. For any $\varepsilon>0$, if $p-1$ has at most $(0.5-\varepsilon) \log \log p$ distinct prime divisors, then $g(p)=o\left(p^{1 / 4}\right)$ as $p \rightarrow \infty$. 
The counting function of primes satisfying the hypothesis of Corollary 2 is $x(\log x)^{-3 / 2+(\log 2) / 2-O(\varepsilon)}$ (the upper bound follows from e.g., [4, Inequality (5)]; the lower bound can be obtained using sieve methods).

Remark 1. The focus of our arguments is to establish bounds which are uniform in $r$. We have made no attempt to optimize the value of $c_{r}$ for small $r$, and leave this as a problem for further study.

Our proof of Theorem 1 proceeds in three main steps. The first is a standard application of character sums to show that a large proportion of integers $n<p^{1 / 4+o(1)}$ are simultaneous $p_{1}, \ldots, p_{r}$-th power nonresidue modulo $p$. Next, we show that if such a number $n$ has many divisors $\left(r 2^{r}\right.$ divisors suffice), then for some pair $d<d^{\prime}$ of these divisors, the smaller number $n^{\prime}=d n / d^{\prime}$ is also a simultaneous $p_{1}, \ldots, p_{r}$-th power nonresidue modulo $p$. This procedure is most efficient when the ratios $d^{\prime} / d$ are uniformly large. In the third step we show that integers possessing many well-spaced divisors are sufficiently dense, so that there must be one such number in the set guaranteed by first step (with an appropriate quantification of "well-spaced" and "dense").

\section{Character sums and distribution of power nonresidues}

We begin by recalling the well-known character sum estimate of Burgess [2, 3].

Lemma 1. If $p$ is a prime and $\chi$ is a non-principal character modulo $p$ and if $H$ and $m$ are arbitrary positive integers, then

$$
\left|\sum_{n=N+1}^{N+H} \chi(n)\right| \ll H^{1-1 / m} p^{(m+1) / 4 m^{2}}(\log p)^{1 / m}
$$

for any integer $N$, where the implied constant is absolute.

See the proof in [11], (12.58). In the remark after the proof the authors announce that the factor $(\log p)^{1 / m}$ can be replaced by $(\log p)^{1 /(2 m)}$, but this is not important for us. 
Lemma 2. Let $p$ be a prime number and $p_{1}, \ldots, p_{r}$ be distinct prime divisors of $p-1$. The number $J$ of integers $n \leqslant H$ which are simultaneous $p_{1}, \ldots, p_{r}$ th power nonresidues modulo $p$ satisfies

$$
J \geqslant 0.12 H \prod_{i=1}^{r}\left(1-\frac{1}{p_{i}}\right)+O\left(r^{13} H^{1-1 / m} p^{(m+1) / 4 m^{2}}(\log p)^{1 / m}\right),
$$

where the constant implied in the "O"-symbol is absolute.

Proof. We follow the method of [5]. Let $C$ be a sufficiently large constant, to be chosen later. Assuming that $p_{1}<\cdots<p_{r}$, we choose the largest $s \leqslant r$ so that $p_{s} \leqslant C r^{2}$ (if $p_{1}>C r^{2}$, then set $s=0$ ). Let $J_{1}$ be the number of integers $n \leqslant H$ which are simultaneous $p_{1}, \ldots, p_{s}$-th power nonresidues modulo $p$. For $j>s$, let $J_{2, j}$ be the number of integers $n \leqslant H$ which are $p_{j}$-th power residues modulo $p$. Clearly,

$$
J \geqslant J_{1}-\sum_{j=s+1}^{r} J_{2, j}
$$

Let $g$ be a primitive root of $p$ and let $\chi_{0}$ be the principal Dirichlet character modulo $p$. We will denote by $\chi$ a generic Dirichlet character modulo $p$. By orthogonality, for $(x, p)=1$ we have

$$
\frac{1}{d} \sum_{\chi^{d}=\chi_{0}} \chi(x)= \begin{cases}1, & \text { if } \operatorname{ind}_{g} x \equiv 0(\bmod d), \\ 0, & \text { if } \operatorname{ind}_{g} x \neq 0(\bmod d) .\end{cases}
$$

A number $n$ is a $p_{i}$-power residue modulo $p$ if and only if $p_{i} \mid \operatorname{ind}_{g} n$. Hence,

$$
J_{1}=\sum_{\substack{n \leqslant H \\ \operatorname{gcd}\left(\operatorname{ind}_{g} n, p_{1} \ldots p_{s}\right)=1}} 1=\sum_{d \mid p_{1} \ldots p_{s}} \mu(d) \sum_{\substack{n \leqslant H \\ d \mid \text { ind }_{g} n}} 1
$$

and for $j=s+1, \ldots, r$ we have

$$
J_{2, j}=\sum_{\substack{n \leqslant H \\ p_{j} \mid \text { ind }_{g} n}} 1
$$

We denote

$$
R=H^{1-1 / m} p^{(m+1) / 4 m^{2}}(\log p)^{1 / m} .
$$


Using Lemma 1 for $\chi \neq \chi_{0}$, we get for any $d$ that

$$
\sum_{\substack{n \leqslant H \\ d \mid \text { ind }_{g} n}} 1=\frac{1}{d} \sum_{\chi^{d}=\chi_{0}} \sum_{n \leqslant H} \chi(n)=\frac{H}{d}+O(R) .
$$

To estimate $J_{1}$ we use a lower bound sieve as in [5] combining with (2.3). Brun's sieve [8, Theorem 2.1 and the following Remark 2] suffices. Here the "sieve dimension" is $\kappa=1$. Taking $\lambda=\frac{1}{4}, b=1, z=C r^{2}$ and $L=O(R)$ in [8, Theorem 2.1 and the following Remark 2], we get that

$$
\begin{aligned}
J_{1} & \geqslant H \prod_{i=1}^{s}\left(1-\frac{1}{p_{i}}\right)\left(1-2 \frac{\lambda^{2 b} e^{2 \lambda}}{1-\lambda^{2} e^{2+2 \lambda}}+O\left(\frac{1}{\log z}\right)\right)-O\left(z^{4.1} R\right) \\
& \geqslant 0.13 H \prod_{i=1}^{s}\left(1-\frac{1}{p_{i}}\right)-O\left(r^{13} R\right)
\end{aligned}
$$

if $C$ is large enough.

By (2.2) and (2.3),

$$
\sum_{j=s+1}^{r} J_{2, j}=H \sum_{j=s+1}^{r} \frac{1}{p_{j}}+O(r R) \leqslant \frac{H}{C r}+O(r R),
$$

since $p_{j}>C r^{2}$ for all $j \geqslant s+1$. Invoking (3.3) and assuming that $C \geqslant 100$, we get

$$
J_{1}-\sum_{j=s+1}^{r} J_{2, j} \geqslant 0.12 H \prod_{i=1}^{s}\left(1-\frac{1}{p_{i}}\right)+O\left(r^{13} R\right) .
$$

Using (2.1) we complete the proof of the lemma.

\section{Reduction of simultaneous nonresidues}

The aim of this section is to show that if a positive integer $n$ which is a simultaneous $p_{1}, \ldots, p_{r}$-th power nonresidue modulo $p$ has many divisors then it is possible to construct $n^{\prime}<n$ which is also a simultaneous $p_{1}, \ldots, p_{r}$-th power nonresidue modulo $p$.

Lemma 3. Let a be a non-zero real number, $\ell \in \mathbb{N}$ and

$$
a_{1}, a_{2}, \ldots, a_{2 \ell-1}
$$


be any sequence of $2 \ell-1$ real numbers (not necessarily distinct). Then for some indices $i_{1}<i_{2}<\ldots<i_{\ell}$ we have that $a_{i_{s}}-a_{i_{t}} \neq$ a for all $1 \leqslant s, t \leqslant \ell$.

Proof. We may assume that $a>0$. Define an equivalence relation on the numbers $i$ by setting $i \sim j$ if $a_{i}-a_{j}=k a$ for some integer $k$. Let $S_{1}, \ldots, S_{m}$ be the different (nonempty) equivalence classes. Clearly $a_{i}-a_{j}=a$ is only possible for $i, j$ within a given equivalence class. Let $b_{r}$ be the smallest element of $S_{r}$, for each $r=1, \ldots, m$. Divide each $S_{r}$ into two subclasses,

$$
\begin{aligned}
& S_{r}^{(0)}=\left\{i \in S_{r}: a_{i}-a_{b_{r}}=k a \text { for some even integer } k\right\}, \\
& S_{r}^{(1)}=\left\{i \in S_{r}: a_{i}-a_{b_{r}}=k a \text { for some odd integer } k\right\} .
\end{aligned}
$$

Obviously $a_{i}-a_{j}=a$ is impossible within each subclass $S_{r}^{(0)}, S_{r}^{(1)}$. For $1 \leqslant r \leqslant m$, define $\varepsilon_{r}=0$ if $\left|S_{r}^{(0)}\right| \geqslant\left|S_{r}^{(1)}\right|$, and $\varepsilon_{r}=1$ otherwise, and let $B=\bigcup_{r=1}^{m} S_{r}^{\left(\varepsilon_{r}\right)}$. Then $|B| \geqslant \ell$, and $a_{i}-a_{j} \neq a$ for $i, j \in B$. Any set $\left\{i_{1}, \ldots, i_{\ell}\right\} \subset B$ then satisfies the requirements of the lemma.

Remark 2. The conclusion of Lemma 3 is best possible, as may be seen by taking $a_{i}=$ ai for $1 \leqslant i \leqslant 2 \ell-1$; in any set of $\ell+1$ elements $a_{i}$ there are two with difference $a$.

Lemma 4. Let $q$ be a prime, $u \in \mathbb{R}, u>1$ and $a \in \mathbb{Z}, a \neq \equiv 0(\bmod q)$. Assume that

$$
a_{1}, a_{2}, \ldots, a_{t}
$$

is a sequence of $t \geqslant 2 u q /(q-1)$ integers (not necessarily distinct). Then for some $\ell \in \mathbb{N}, \ell \geqslant u$ and indices $i_{1}<i_{2}<\ldots<i_{\ell}$ we have that

$$
a_{i_{v}}-a_{i_{w}} \not \equiv a(\bmod q) \quad(1 \leqslant v, w \leqslant \ell) .
$$

Proof. We can assume that $a=1$. Define $\ell=\lceil u\rceil$. From the pigeon-hole principle, there is a residue class $h(\bmod q)$ containing at most $t / q$ elements from the sequence (3.2). Since

$$
\left\lceil t-\frac{t}{q}\right\rceil=\lceil t(q-1) / q\rceil \geqslant\lceil 2 u\rceil \geqslant 2 \ell-1
$$

after rearranging (3.2) we may assume that

$$
a_{s} \not \equiv h(\bmod q) \quad(s=1,2, \ldots, 2 \ell-1) .
$$


Define $c_{s} \in\{1,2, \ldots, q-1\}$ by

$$
c_{s} \equiv a_{s}-h(\bmod q) .
$$

By Lemma 3, there is a subsequence $c_{i_{1}}, \ldots, c_{i_{\ell}}$ such that

$$
c_{i_{v}}-c_{i_{w}} \neq 1 \quad(1 \leqslant v, w \leqslant \ell) .
$$

Since $1 \leqslant c_{i} \leqslant q-1$ this implies that

$$
c_{i_{v}}-c_{i_{w}} \not \equiv 1(\bmod q) \quad(1 \leqslant v, w \leqslant \ell)
$$

and thus

$$
a_{i_{v}}-a_{i_{w}} \not \equiv 1(\bmod q) \quad(1 \leqslant v, w \leqslant \ell) .
$$

Remark 3. For $q=2$ it is enough to require $t \geqslant 2 u$. Indeed, we can choose a large subsequence of $a_{1}, a_{2}, \ldots, a_{t}$ of the same parity.

Corollary 3. Let $p_{1}, p_{2}, \ldots, p_{r}$ be prime numbers, and

$$
\mathbf{b}=\left(b_{1}, b_{2}, \ldots, b_{r}\right) \in \mathbb{F}_{p_{1}}^{*} \times \mathbb{F}_{p_{2}}^{*} \times \ldots \times \mathbb{F}_{p_{r}}^{*} .
$$

Let

$$
t>2^{r} \prod_{i: p_{i}>2} \frac{p_{i}}{p_{i}-1}
$$

and

$$
\mathbf{a}_{1}, \mathbf{a}_{2} \ldots, \mathbf{a}_{t}
$$

be a sequence of $t$ elements from $\mathbb{F}_{p_{1}} \times \mathbb{F}_{p_{2}} \times \ldots \times \mathbb{F}_{p_{r}}$. Then for some $i<j$ we have that

$$
\mathbf{a}_{j}-\mathbf{a}_{i} \in\left(\mathbb{F}_{p_{1}} \backslash\left\{b_{1}\right\}\right) \times\left(\mathbb{F}_{p_{2}} \backslash\left\{b_{2}\right\}\right) \times \ldots \times\left(\mathbb{F}_{p_{r}} \backslash\left\{b_{r}\right\}\right) .
$$

Corollary 3 follows from $r$ applications of Lemma 4 and taking into account Remark 3 .

Corollary 4. Let $p$ be a prime number and suppose $p_{1}, \ldots, p_{r}$ are distinct prime divisors of $p-1$. Let $n$ be a simultaneous $p_{1}, \ldots, p_{r}$-th power nonresidue modulo $p$ and $d_{1}<\cdots<d_{t}$ be some divisors of $n$ where

$$
t>2^{r} \prod_{p_{i}>2} \frac{p_{i}}{p_{i}-1} .
$$

Then there exists $i, j$ such that $1 \leqslant i<j \leqslant t$ and the number $n^{\prime}=n d_{i} / d_{j}$ is also a simultaneous $p_{1}, \ldots, p_{r}$-th power nonresidue modulo $p$. 
Proof. Let $g$ be a primitive root modulo $p$. To each number $x$ we associate the vector

$$
\left(u_{1}, u_{2}, \ldots, u_{r}\right) \in \mathbb{F}_{p_{1}} \times \mathbb{F}_{p_{2}} \times \ldots \times \mathbb{F}_{p_{r}},
$$

so that for $1 \leqslant i \leqslant r, x \equiv g^{p_{i} k_{i}+s_{i}}(\bmod p)$ where $0 \leqslant s_{i}<p_{i}$

Let the vector $\left(b_{1}, b_{2}, \ldots, b_{r}\right)$ correspond to $n$ and the vectors $\mathbf{a}_{1}, \mathbf{a}_{2}, \ldots, \mathbf{a}_{t}$ correspond to $d_{1}, \ldots, d_{t}$, respectively. Apply Corollary 3 and select the indices $i<j$ such that

$$
\mathbf{a}_{j}-\mathbf{a}_{i} \in\left(\mathbb{F}_{p_{1}} \backslash\left\{b_{1}\right\}\right) \times\left(\mathbb{F}_{p_{2}} \backslash\left\{b_{2}\right\}\right) \times \ldots \times\left(\mathbb{F}_{p_{r}} \backslash\left\{b_{r}\right\}\right)
$$

Then $n^{\prime}=n d_{i} / d_{j}$ is a simultaneous $p_{1}, p_{2}, \ldots, p_{r}$-power nonresidue modulo $p$.

Remark 4. We note that if $p_{1}, p_{2}, \ldots, p_{r}$ are distinct primes, then

$$
r>\prod_{p_{i}>2} \frac{p_{i}}{p_{i}-1}
$$

Hence, in Corollaries 3 and 4 one can take $t=2^{r} r$.

\section{Integers with well-spaced divisors}

Let $P^{-}(n)$ and $P^{+}(n)$ denote the smallest and largest prime factor of $n$, respectively, let $\omega(n)$ be the number of distinct prime factors of $n$, and let $\tau(n)$ be the number of positive divisors of $n$.

Lemma 5. For each fixed constant $c>1 / \log 2=1.442 \ldots$, there is $\eta=$ $\eta(c)>0$ such that the following holds. Uniformly for integers $t, 2 \leqslant t \leqslant$ $(\log x)^{1 / c}$, all but $O_{c}\left(x / t^{\eta}\right)$ integers $n \leqslant x$ have $t$ divisors $d_{1}<d_{2}<\cdots<d_{t}$ such that $d_{j+1} / d_{j}>x^{1 / t^{c}}$ for all $1 \leqslant j \leqslant t-1$.

Proof. We may assume that $t \geqslant 10$. Take

$$
\varepsilon=\frac{c-1 / \log 2}{4}, \quad \alpha=1 / \log 2+\varepsilon .
$$

Write each $n \leqslant x$ in the form abd where $P^{-}(d)>x^{1 / \log t}, P^{+}(a) \leqslant x^{1 /\left(t^{\alpha} \log t\right)}$ and all prime factors of $b$ lie in $\left(x^{1 /\left(t^{\alpha} \log t\right)}, x^{1 / \log t}\right]$. We divide $n$ into several categories. Let $k_{0}=\left\lceil\frac{\log 2 t}{\log 2}\right\rceil$. Let $S_{0}$ be the set of $n \leqslant x$ with either $d=1$ 
or with $b$ not squarefree. Let $S_{1}$ be the set of $n$ with $d>1, b$ squarefree and $\omega(b)<k_{0}$. We denote $\alpha_{j}=j \varepsilon$ for $1 \leqslant j \leqslant J-1:=[\alpha / \varepsilon], \alpha_{J}=\alpha$, $a_{j}=x^{1 /\left(t^{\alpha_{j}} \log t\right)}$ for $j=1, \ldots, J$. Let $S_{2}$ be the set of $n$ with $d>1, b$ squarefree and the number of primes from the interval $\left(a_{j}, x^{1 / \log t}\right]$ dividing $n$ is less than $k_{j}:=\left(\alpha_{j}-\varepsilon\right) \log t$ for some $j=1, \ldots, J-1$. Let $S_{3}$ be the set of the remaining integers $n$.

We first show that $S_{0}, S_{1}$, and $S_{2}$ are small. By standard counts for smooth numbers,

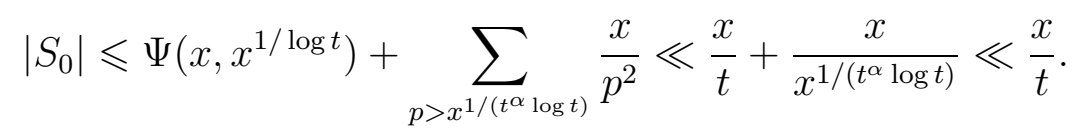

Next, by the results of Halász [7] on the number of integers with a prescribed number of prime factors from a given set (see also Theorem 08 of [10]), we have

$$
\begin{aligned}
\left|S_{1}\right| & \ll \sum_{k<k_{0}} x e^{-E} \frac{E^{k}}{k !}, \quad E=\sum_{x^{1 /\left(t^{\alpha} \log t\right)<p \leqslant x^{1 / \log t}} \frac{1}{p}=\alpha \log t+O(1)} \\
& \ll x t^{-\alpha} \sum_{k<k_{0}} \frac{(\alpha \log t)^{k}}{k !} \\
& \ll x\left(t^{\alpha}\right)^{-(\beta \log \beta-\beta+1)}, \quad \beta=\frac{1}{\alpha \log 2}=\frac{1}{1+\varepsilon \log 2}<1 \\
& \ll x / t^{\delta}
\end{aligned}
$$

for some $\delta>0$ which depends on $\varepsilon$.

For any $j=1, \ldots, J-1$ we denote by $S_{2, j}$ the set of $n \leqslant x$ with less than $k_{j}$ prime divisors from $\left(a_{j}, x^{1 / \log t}\right]$. We have

$$
\left|S_{2, j}\right| \ll \sum_{k<k_{j}} x e^{-E_{j}} \frac{E_{j}^{k}}{k !},
$$

where

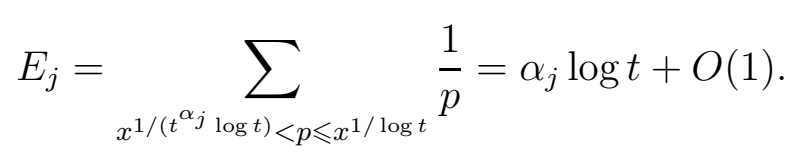

Arguing as before we get

$$
\left|S_{2, j}\right| \ll x / t^{\delta^{\prime}}
$$


for some $\delta^{\prime}>0$ which depends on $\varepsilon$.

Notice that for $n \in S_{3}, \tau(b)=2^{\omega(b)} \geqslant 2^{k_{0}} \geqslant 2 t$. Next, let $S_{4}$ be the set of $n \in S_{3}$ for which $b$ does not have $t$ well-spaced divisors in the sense of the lemma. Since $d>1$ for such $n$, given such a bad value of $b$, using a standard sieve bound the number of choices for the pair $(a, d)$ is bounded above by

$$
\sum_{a}\left|\left\{d \leqslant x / a b: P^{-}(d)>x^{1 / \log t}\right\}\right| \ll \sum_{a} \frac{x / a b}{\log \left(x^{1 / \log t}\right)} \ll \frac{x}{b t^{\alpha}} .
$$

Hence,

$$
\left|S_{4}\right| \ll \sum_{\operatorname{bad} b} \frac{x}{b t^{\alpha}}
$$

A number $b$ which is bad has many pairs of neighbor divisors. To be precise, let $\sigma=t^{-c} \log x$ and define

$$
W^{*}(b ; \sigma)=\left|\left\{\left(d^{\prime}, d^{\prime \prime}\right): d^{\prime}\left|b, d^{\prime \prime}\right| b, d^{\prime} \neq d^{\prime \prime},\left|\log \left(d^{\prime} / d^{\prime \prime}\right)\right| \leqslant \sigma\right\}\right| .
$$

Let $d_{1}<\cdots<d_{\tau(b)}$ be the divisors of $b$. We construct the subsequence $D_{1}<\cdots<D_{r}$ of this sequence:

$$
D_{1}=1, \quad D_{i}=\min \left\{d_{j}: d_{j}>x^{t^{-c}} D_{i-1}\right\}(i>1) .
$$

The process is terminated if $D_{i}$ does not exist. Let $D_{r+1}=+\infty$. The set $\left\{d_{1}, \ldots, d_{\tau(b)}\right\}$ is divided into $r$ subsets $\mathcal{D}_{i}, i=1, \ldots, r$, where

$$
\mathcal{D}_{i}=\left\{d_{j}: D_{i} \leqslant d_{j}<D_{i+1}\right\}
$$

We see that $\left(d^{\prime}, d^{\prime \prime}\right)$ is counted in $W^{*}(b ; \sigma)$ if $d^{\prime}, d^{\prime \prime} \in \mathcal{D}_{i}$ for some $i$ and $d^{\prime} \neq d^{\prime \prime}$. Hence,

$$
W^{*}(b ; \sigma) \geqslant \sum_{i=1}^{r}\left|\mathcal{D}_{i}\right|\left(\left|\mathcal{D}_{i}\right|-1\right)=\sum_{i=1}^{r}\left|\mathcal{D}_{i}\right|^{2}-\tau(b)
$$

Since $\tau(b) \geqslant 2 t$ and $r \leqslant t$, we get by the Cauchy-Schwartz inequality that

$$
\tau(b)^{2}=\left(\sum_{i=1}^{r}\left|\mathcal{D}_{i}\right|\right)^{2} \leqslant t\left(\sum_{i=1}^{r}\left|\mathcal{D}_{i}\right|^{2}\right) \leqslant t\left(W^{*}(b ; \sigma)+\tau(b)\right) \leqslant t W^{*}(b ; \sigma)+\frac{1}{2} \tau(b)^{2} .
$$

Therefore,

$$
\sum_{\operatorname{bad} b} \frac{1}{b} \leqslant \sum_{\text {all } b} \frac{2 W^{*}(b ; \sigma) t}{b \tau(b)^{2}}
$$


each sum being over squarefree integers whose prime factors lie in $\left(x^{1 /\left(t^{\alpha} \log t\right)}, x^{1 / \log t}\right]$.

In the latter sum, fix $k=\omega(b)$, write $b=p_{1} \cdots p_{k}$, where the $p_{i}$ are primes, and $p_{1}<\cdots<p_{k}$. Then $W^{*}\left(p_{1} \cdots p_{k} ; \sigma\right)$ counts the number of pairs $Y, Z \subset\{1, \ldots, k\}$ with $Y \neq Z$ and

$$
\left|\sum_{i \in Y} \log p_{i}-\sum_{i \in Z} \log p_{i}\right| \leqslant \sigma .
$$

Fix $Y, Z$, and let $I$ be the maximum element of the symmetric difference $(Y \cup Z)-(Y \cap Z)$. We fix $I$ and count the number of $p_{1}, \ldots, p_{k}$ satisfying (4.3). We further partition the solutions, according to the condition $a_{j}<p_{I} \leqslant a_{j-1}$, for $j=1, \ldots, J$. Fix the value of $j$. If all the $p_{i}$ are fixed except for $p_{I}$, then (4.3) implies that $p_{I}$ lies in some interval of the form $\left[U, U e^{2 \sigma}\right]$. As $p_{I}>x^{1 / t^{\alpha_{j}} \log t}$ as well, and $\alpha>c$, we have (putting $U_{j}=\max \left(U, x^{1 / t^{\alpha_{j}} \log t}\right)$ )

$$
\sum_{p_{I}} \frac{1}{p_{I}} \ll \log \left(1+\frac{2 \sigma}{\log U_{j}}\right) \ll \frac{\sigma}{\log U_{j}} \ll t^{\alpha_{j}-c} \log t
$$

Hence, for each fixed $k, j, Y$ and $Z$,

$$
\begin{aligned}
\sum_{x^{1 / t^{\alpha} \log t}<p_{1}<\ldots<p_{k} \leqslant x^{1 / \log t}} \frac{1}{p_{1} \cdots p_{k}} & \ll \frac{t^{\alpha_{j}-c}(\log t)}{(k-1) !}\left(\sum_{\left.x^{1 / t^{\alpha} \log t<p \leqslant x^{1 / \log t}} \frac{1}{p}\right)^{k-1}}\right. \\
& \ll \frac{t^{\alpha_{j}-c}(\log t)(\alpha \log t+O(1))^{k-1}}{(k-1) !} .
\end{aligned}
$$

Now we estimate the number $N(I, j)$ of choices for the pair $Y, Z$ for fixed $I$ and $j$. Since $p_{I} \leqslant a_{j-1}$, the condition $n \in S_{3}$ implies $I \leqslant k-k_{j-1}$. For any $i \leqslant I$ there are at most four possibilities: $i \in Y \cap Z, i \in Y \backslash Z, i \in Z \backslash Y$, $i \notin Y \cup Z$. For $i>I$ there are two possibilities: $i \in Y \cap Z$ and $i \notin Y \cup Z$. Therefore,

$$
N(I, j) \leqslant 4^{I} 2^{k-I} \leqslant 4^{k} 2^{-k_{j-1}} \leqslant 4^{k} t^{-\alpha_{j} \log 2+2 \varepsilon \log 2} .
$$

It follows from (4.4) and (4.5) that

$$
\sum_{\omega(b)=k} \frac{W^{*}(b ; \sigma) t}{b \tau(b)^{2}} \ll \sum_{j=1}^{J} t^{1+(1-\log 2) \alpha_{j}+2 \varepsilon-c} \sum_{k} \frac{(\alpha \log t+O(1))^{k-1}}{(k-1) !}
$$


Taking into account that $\alpha_{j} \leqslant \alpha$ and summing on $j, k$ we get

$$
\sum_{b} \frac{W^{*}(b ; \sigma) t}{b \tau(b)^{2}} \ll t^{1+2 \varepsilon+(2-\log 2) \alpha-c} .
$$

Thus, by (4.1) and (4.2),

$$
\left|S_{4}\right| \ll \frac{x}{t^{c-(1-\log 2) \alpha-2 \varepsilon-1}}=\frac{x}{t^{c-1 / \log 2-\varepsilon(3-\log 2)}} \ll \frac{x}{t^{\varepsilon}} .
$$

Therefore, there are $x-O\left(x / t^{\min \left(\delta, \delta^{\prime}, \varepsilon\right)}\right)$ numbers $n \leqslant x$ for which $b$ does have $t$ well-spaced divisors.

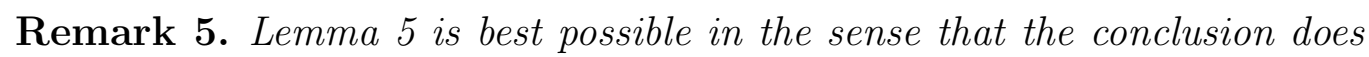
not hold for $c<1 / \log 2$. In fact, for any $c<1 / \log 2$, the number of integers $n \leqslant x$ that do have $t$ divisors $d_{1}, \ldots, d_{t}$ with $d_{j+1} / d_{j}<n^{1 / t^{c}}$ for all $j$ is $O_{c}\left(x / t^{\eta}\right)$ for some $\eta>0$ which depends on $c$.

Proof. It is well-known that if $t$ is large, $c<1 / \log 2$ and $\varepsilon$ small enough, then a typical integer $n$ has $r \sim(c+\varepsilon) \log t$ prime factors $p_{1}, \ldots, p_{r}$ in $\left[n^{1 / t^{c+\varepsilon}}, n\right]$. This can be seen, e.g. by the theorem of Halász used in the estimation of $\left|S_{1}\right|$. In fact, the number of exceptional $n \leqslant x$ is $O_{c}\left(x / t^{\eta}\right)$. Thus, a typical $n$ has about $2^{(c+\varepsilon) \log t}=t^{(c+\varepsilon) \log 2}<t$ divisors composed of such primes. Also, for most of these $n, n /\left(p_{1} \cdots p_{r}\right)<n^{1 /\left(2 t^{c}\right)}$; by Theorem 07 of [10], the number of exceptions $n \leqslant x$ is $O\left(x \exp \left\{-c_{1} t^{\varepsilon}\right\}\right)$ for some $c_{1}>0$. Suppose that such an $n$ has $t$ well-spaced divisors $d_{1}, \ldots, d_{t}$ with $d_{j+1} / d_{j}<n^{1 / t^{c}}$ for all $j$. By the pigeon-hole principle, two of these divisors share the same set of prime factors from $\left\{p_{1}, \ldots, p_{r}\right\}$, hence their ratio is less than $n^{1 /\left(2 t^{c}\right)}$, a contradiction.

\section{Proof of Theorem 1}

We rewrite the assertion of Lemma 2 as

$$
J \geqslant 0.12 H \prod_{i=1}^{r}\left(1-\frac{1}{p_{i}}\right)-R^{\prime}, \quad R^{\prime}=(5 r)^{C^{\prime \prime}} H^{1-1 / m} p^{(m+1) / 4 m^{2}}(\log p)^{1 / m}
$$

for some constant $C^{\prime \prime}$. Let $\mathcal{N}$ denotes the set of $n \in[1, H]$ which are simultaneous $p_{1}, \ldots, p_{r}$-th power nonresidue modulo $p$, where

$$
H=p^{1 / 4} e^{\left(C^{\prime \prime}+3\right)(\log p)^{1 / 2}(\log (5 r))^{1 / 2}} \log p .
$$


Assume that $p$ is sufficiently large, and take

$$
m=\left\lfloor(\log p)^{1 / 2}(\log (5 r))^{-1 / 2}\right\rfloor .
$$

Notice that $m \gg(\log p)^{1 / 2}(\log \log p)^{-1 / 2} \rightarrow \infty$ as $p \rightarrow \infty$. Since

$$
R^{\prime}=H\left(H p^{-1 / 4} / \log p\right)^{-1 / m} p^{1 /\left(4 m^{2}\right)}(5 r)^{C^{\prime \prime}},
$$

we have

$$
\left(H p^{-1 / 4} / \log p\right)^{-1 / m} \leqslant(5 r)^{-C^{\prime \prime}-3}
$$

and

$$
p^{1 /\left(4 m^{2}\right)} \leqslant 5 r .
$$

Consequently,

$$
R^{\prime} \leqslant H(5 r)^{-2}
$$

By (5.1) and (3.3),

$$
J \geqslant\left(0.12 r^{-1}-(5 r)^{-2}\right) H \geqslant 0.08 H / r .
$$

So, we see that

$$
|\mathcal{N}| \geqslant 0.08 H / r
$$

We consider the case

$$
r<0.6 \log \log p
$$

first. We will apply Lemma 5 with $x=H$, fixed $c \in(1 / \log 2,1.5]$, and with $t=K r 2^{r}$, where $K$ is a sufficiently large constant depending on $c$. By (5.2), the exceptional set in Lemma 5 is smaller than $|\mathcal{N}|$ provided that $K$ is large enough. The condition $2 \leqslant t \leqslant(\log x)^{1 / c}$ is satisfied due to the restriction on $r$ and $c$. By Lemma 5, for some $n \in \mathcal{N}$, there are well-separated divisors $d_{1}<\cdots<d_{t}$ of $n$, satisfying $d_{i+1} / d_{i}>n^{1 / t^{c}}$ for each $i$. Now we are in position to apply Corollary 4 and we see that there is an $n^{\prime} \leqslant n p^{-t^{-c} / 4}$ such that $n^{\prime}$ is a simultaneous $p_{1}, \ldots, p_{r}$-th power nonresidue modulo $p$. Noting that $t^{-c} / 4=\exp \{-r(c \log 2+o(1))\}$ and that $c$ may be taken arbitrarily close to $1 / \log 2$, we complete the proof.

If (5.3) does not hold, then, as we have mentioned in Section 1, the factor $p^{-c_{r}}$ in the statement of the theorem is dominated by the second factor, and the claim follows from the fact that $\mathcal{N} \neq \emptyset$. 


\section{Acknowledgements}

The first author is supported in part by National Science Foundation grants DMS-1201442 and DMS-1501982. The third author is supported by grant RFBR 14-01-00332 and grant Leading Scientific Schools N 3082.2014.1.

\section{References}

[1] D. A. Burgess, 'The distribution of quadratic residues and nonresidues', Mathematika, 4 (1957), 106-112.

[2] D. A. Burgess, 'On character sums and primitive roots', Proc. London Math. Soc., 12 (1962), 179-192.

[3] D. A. Burgess, 'On character sums and L-series. II.', Proc. London Math. Soc., 13 (1963), 524-536.

[4] P. Erdős, 'On the normal number of prime factors of $p-1$ and some related problems concerning Euler's $\varphi$-function', Quart. J. Math. Oxford Ser., 6 (1935), 205-213.

[5] P. Erdős, 'On the least primitive root of a prime', Bull. London Math. Soc., 55 (1945), 131-132.

[6] P. Erdős, H. N. Shapiro, 'On the least primitive root of a prime', Pacific J. Math., 7 (1957), 861-865.

[7] G. Halász, 'Remarks to my paper: "On the distribution of additive and the mean values of multiplicative arithmetic functions", Acta Math. Acad. Sci. Hungar., 23 (1972), 425-432.

[8] H. Halberstam, H.-E. Richert, Sieve methods, Academic Press, 1974.

[9] L.-K. Hua, 'On the least primitive root of a prime', Bull. Amer. Math. Soc., 48 (1942), 726-730.

[10] R. R. Hall, G. Tenenbaum, Divisors, Cambridge Tracts in mathematics vol. 90, 1988.

[11] H. Iwaniec, E. Kowalski, Analytic number theory, American Mathematical Society, Providence, Rhode Island, 2004. 
[12] Yu. V. Linnik, 'A remark on the least quadratic non-residue', (Russian) C. R. (Doklady) Acad. Sci. URSS (N.S.), 36 (1942), 119-120.

[13] G. Martin, 'The least prime primitive root and the shifted sieve', Acta Arith., 80 (1997), no. 3, 277-288.

[14] I. M. Vinogradov, 'On the distribution of quadratic residues and nonresidues', (in Russian), Journal of the Physico-Mathematical Society of Perm, 1919.

[15] I. M. Vinogradov, 'On the least primitive root', (Russian), Doklady Akad. Nauk SSSR, 1 (1930), 7-11.

Address of the authors:

K. Ford, Department of Mathematics, 1409 West Green Street, University of Illinois at Urbana-Champaign, Urbana, IL 61801, USA.

E-mail address: ford@math.uiuc.edu

M. Z. Garaev, Centro de Ciencias Matemáticas, Universidad Nacional Autónoma de México, C.P. 58089, Morelia, Michoacán, México.

Email address: garaev@matmor.unam.mx

S. V. Konyagin, Steklov Mathematical Institute, 8 Gubkin Street, Moscow, 119991, Russia.

Email address: konyagin@mi.ras.ru 